\title{
Evaluación del control de calidad de la carne vacuna consumida en la ciudad de Pilar, año 2017
}

\author{
Alberto Andrés Ruíz Gómez \\ ingagpalbertoruiz@gmail.com \\ Carlos Eliezer Benítez González \\ carloshuevito@hotmail.com \\ César Rolando dos Santos Pavón \\ Cesarito2s@hotmail.com \\ Universidad Nacional de Pilar. \\ Ciudad de Pilar - Paraguay.
}

\section{RESUMEN}

Se considera, a la carne, el tejido animal más apropiado para ser usado como alimento. Por ello, este estudio se realiza con el objetivo de caracterizar las condiciones higiénicosanitarias del proceso transporte y comercialización de la carne vacuna en la ciudad de Pilar, año 2017. Adoptando como estrategia metodológica un enfoque cuantitativo y cualitativo, con diseño observacional, transversal y prospectivo, a partir de la administración de un cuestionario a los propietarios de las carnicerías de la ciudad de Pilar, una Entrevista semiestructurada a responsables del control de calidad de la Municipalidad local y representantes del SENACSA de la ciudad de Pilar, el Análisis Documental, con la revisión de registros históricos, solicitados y extraídos de los entes encargados de la calidad y sanidad animal; además de la Observación directa. La población se compone por 64 carnicerías de la ciudad de Pilar y la muestra seleccionada en la elección al azar del 30\% de las carnicerías de Pilar, asciende a 20 negocios; se puede mencionar que es recular la situación del transporte y comercialización de carne vacuna en la ciudad de Pilar, haciendo la salvedad de que falta un mayor control del ingreso y procedencia de las carnes comercializadas en determinadas carnicerías.

Palabras clave: evaluación; carne; control; calidad; transporte. 


\title{
Evaluation of quality control of beef consumed in the city of Pilar, year 2017
}

\begin{abstract}
Meat is considered the most appropriate animal tissue to be used as food. For this reason, this study is carried out with the objective of characterizing the hygienic-sanitary conditions of the transport and commercialization process of beef in the city of Pilar, year 2017. Adopting as a methodological strategy a quantitative and qualitative approach, with an observational, cross-sectional design and prospective, from the administration of a questionnaire to the owners of the butcher shops in the city of Pilar, a semi-structured interview with those responsible for quality control of the local Municipality and representatives of the SENACSA of the city of Pilar, the Documentary Analysis, with the review of historical records, requested and extracted from the entities in charge of quality and animal health; in addition to direct observation. The population is made up of 64 butcher shops in the city of Pilar and the sample selected in the random choice of $30 \%$ of the butcher shops in Pilar amounts to 20 businesses; It can be mentioned that it is to reverse the situation of transportation and commercialization of beef in the city of Pilar, with the exception that there is a lack of greater control of the income and origin of the meats sold in certain butcher shops.
\end{abstract}

Keywords: evaluation; meat; control; quality; transport.

Artículo recibido: 30 noviembre. 2021 Aceptado para publicación: 29 diciembre 2021 Correspondencia: ingagpalbertoruiz@gmail.com Conflictos de Interés: Ninguna que declarar 


\section{INTRODUCCIÓN}

La producción bovina y el sector cárnico como industria en Paraguay, han tenido un desarrollo significativo en los últimos años, el crecimiento de la ganadería local se viene dando desde hace 13 años a nivel de entre 5 a 5,5\% en volumen de cantidad.

La calidad de la carne bovina está particularmente definida por su composición química (valor nutricional) y por sus características organolépticas (valor sensorial) tales como la terneza, el color, el sabor y la jugosidad. El sistema de producción, el tipo de animal, el plano nutricional ofrecido $y$ el manejo pre $y$ post faena, pueden modificar considerablemente estas características.

La calidad de los alimentos no está uniformemente definida. Al juzgar la calidad de la carne y de los productos cárnicos el consumidor tiene en cuenta un conjunto de características y no una característica única. Al asignar un grado de importancia a cada una de las características de calidad debe tenerse en consideración el valor "ideal u óptimo" dentro de un marco realista de la rentabilidad en un mercado competitivo. Se ha sugerido que también la uniformidad y la consistencia son importantes componentes de la calidad. La calidad, por tanto, es un concepto relativo que va desde la impresión global que se hace al público de una marca comercial hasta la preferencia personal del consumidor respecto al color, textura y sabor (Hernández Aliaga, 2009). De esta manera el objetivo principal de este estudio es: Caracterizar las condiciones higiénico-sanitarias del proceso transporte y comercialización de la carne vacuna en la ciudad de Pilar, año 2017.

A partir del conocimiento de las disposiciones legales previstas por los Organismos de control de calidad en el proceso de transporte y comercialización de la carne vacuna; la descripción, de las características físicas y sensoriales que presentan las carnes que se expenden en el mercado de la ciudad de Pilar, y el manejo de los mecanismos de control establecidos para la verificación de la calidad de los productos ofrecidos al público por parte de las Carnicerías de la ciudad de Pilar.

Por la cual, se da respuesta a la interrogante: ¿Cuáles son las condiciones higiénicosanitarias del proceso de transporte y comercialización de la carne vacuna en la ciudad de Pilar, en el año 2017? 


\section{Antecedentes}

La industria cárnica posee gran peso en la actividad económica del Paraguay. La producción de carne constituye el subsector más importante dentro del PIB (Asociación Rural del Paraguay, 2017).

Desde el año 2004, la Exportación de "Carne y Despojo Comestible" ha presentado un gran aumento, siendo actualmente el $4^{\circ}$ Rubro de Mayor Exportación Nacional (Unidad Técnica de Estudios para la Industria, 2008). El aumento en el nivel de exportaciones de la carne vacunase debió a 2 situaciones muy particulares ocurrido en Brasil y Argentina, lo que posibilitó a nuestro país ganar nuevos mercados.

Se busca aumentar la cantidad de carne y mejorar su calidad para mantener a los pueblos sanos y bien nutridos. Junto a estos valores intrínsecos de la carne, está el hecho que ella es un producto perecible y puede ser el origen en los seres humanos de graves enfermedades tóxico - infecciosas, si ésta no reúne las mejores condiciones de sanidad, conservación y manejo antes de ser consumida (Grupo Latino Ltda., 2006).

Cuando se habla de la calidad de carnes frescas, algunos de los atributos que el consumidor busca son la terneza, jugosidad y color. Estas propiedades están influenciadas por varios factores como la raza del animal, manejo antemortem, procesos de matanza, el manejo de las canales durante el almacenamiento, comercialización, etc. (San Román, 2015).

Esta investigación enfrenta uno de los principales problemas de la salud pública que existe en la sociedad, dentro de la ciudad de Pilar no existen políticas de observación al reglamento de agro-calidad que permitan determinar las condiciones higiénico-sanitarias en que se realiza el proceso de faenamiento, transporte y comercialización de la carne; si bien es cierto la Municipalidad de la ciudad de Pilar, es la instancia encargada de responder a los requerimientos del resguardo de la calidad de producción y comercialización; sin embargo, es necesario determinar la situación actual, mediante un conjunto de investigaciones para en base a los resultados obtenidos formular una propuesta, que tratará de garantizar la calidad e inocuidad de este alimento básico para los habitantes de la ciudad.

\section{Justificación.}

La calidad es un concepto complejo y difícil de definir. La principal dificultad estriba en que los distintos eslabones de la cadena cárnica (ganadero, matadero, carnicero y 
consumidor) valoran la calidad desde distintos puntos de vista, en función de sus propios objetivos. Además, el concepto de calidad varía ampliamente con la zona geográfica, circunstancias sociales y económicas, incluso varía a lo largo del tiempo. Además, la calidad de la carne puede ser considerada desde distintos puntos de vista: Calidad nutritiva: en función del contenido y proporción de nutrientes; la Calidad higiénica: según la presencia de residuos tóxicos, carga microbiana, etc., y la Calidad tecnológica: depende de la adecuación de la carne a la elaboración de los diversos productos cárnicos (Horcada, 2000).

Calidad sensorial u organoléptica: en función de los atributos sensoriales percibidos bien por la vista (que determinarán la compra o no de la carne) o bien durante el consumo (que determinarán la aceptabilidad final del producto) (Instituto de Nutrición de Centro América y Panamá, 2020).

La importancia de la elaboración de este trabajo de investigación radica fundamentalmente en la necesidad de instaurar un sistema de control de calidad de los productos ofrecidos al público por parte de las Empresas Cárnicas establecidas dentro de la comunidad, en consideración a la existencia de normativas que establecen los procedimientos y los cuidados que deben ser asumidos por los comercios, existiendo hasta el momento escasas informaciones con relación al tema.

Este trabajo permitirá ofrecer a la comunidad un análisis de las condiciones de calidad de los productos consumidos y por ende la determinación de los parámetros que deberán ser aprobados por las instancias correspondientes en el sistema de control y supervisión por parte de las instituciones encargadas.

Se considera, a la carne, el tejido animal más apropiado para ser usado como alimento. Se subdivide en varias categorías generales: carnes rojas y blancas basándose en la concentración del pigmento mioglobina; también en carne de animales de finca (res, cerdos, aves, etc.), mariscos y animales no domesticados. Dentro de la categoría de carnes rojas se identifican la de vacuno, cerdo, cordero y ternera (Hedrick et al., 1994; Aberle et al., 2001).

La carne es un alimento fácilmente digestible y supone una excelente fuente de proteína de alta calidad. Además, se trata de un alimento rico en vitaminas del complejo B y constituye una fuente importante de minerales como el hierro. La ingestión de 100 gramos de carne aporta al organismo de 210 a $250 \mathrm{kcal}$. Considerando las necesidades energéticas 
diarias de un hombre adulto, podemos concluir que la carne es un elemento de gran importancia como aporte energético en nuestra dieta (Tovar, 2019).

\section{Faenamiento del ganado bovino}

Los manejos que se realizan en el ganado destinado a producir carne en horas previas a su sacrificio, son de los más estresantes en su vida y pueden provocar, además, un serio deterioro de la calidad del producto (Warriss, 1990; 1992) citado en (Gallo \& Tadich B., 2008). Dichos manejos tienen importancia desde cuatro puntos de vista esenciales:

- Aspectos éticos: los seres humanos, y especialmente los profesionales del área pecuaria, deben propender a evitar el sufrimiento innecesario de los animales destinados a producir carne para la alimentación humana (Gallo \& Tadich B., 2008).

- Cantidad de carne producida: el transporte inadecuado, los largos tiempos de privación de alimento, así como los malos tratos durante los manejos previos al sacrificio provocan disminuciones de peso en las canales y hematomas (contusiones, lesiones) que implican recortes de trozos de la canal con las consiguientes mermas de peso.

- Calidad de carne producida: el manejo inadecuado en esta etapa provoca estrés en los animales; este estrés conlleva cambios de tipo metabólico y hormonal a nivel muscular en el animal vivo, que se traducen en cambios de color, $\mathrm{pH}$ y capacidad de retención de agua en el músculo postmortem. Como consecuencia de ello, las características de la carne cambian, tornándose menos aceptables al consumidor y acortándose la vida útil del producto.

- Exigencias reglamentarias: en los últimos tiempos existe una creciente preocupación por parte de los consumidores en cuanto a que los animales deben ser producidos bajo estándares de bienestar aceptables y manejados en forma humanitaria durante el beneficio, aspectos que deben ser además registrados en un sistema de trazabilidad del producto, para poder diferenciarlos, citados en (Gallo \& Tadich B., 2008).

Esto ha llevado a un aumento de las exigencias legales y reglamentarias en torno al bienestar animal. El esquema de comercialización utilizado para el ganado bovino, las características de producción y de funcionamiento de las plantas faenadoras, así como las condiciones de manejo antes del faenamiento indican un alto riesgo de problemas de bienestar animal y de calidad de la carne en estas etapas. Cabe destacar entre otros aspectos, que más del $50 \%$ de los bovinos se traslada en pie desde los centros de 
producción a los de consumo; que los tiempos de reposo en ayuno utilizados en las plantas faenadoras, en general superan las 12 horas; que hay una creciente detección de carnes afectadas por problemas debidos al estrés, tal como corte oscuro, y que hay una escasa atención al sufrimiento innecesario ocasionado a los animales durante el arreo y la insensibilización (Gallo, 1997).

Entre los aspectos más importantes para lograr esto y facilitar el arreo de los animales, está el diseño de estructuras adecuadas (mangas, corrales, rampas de carga, cercos, pisos) la eliminación de las llamadas distracciones que impiden un normal avance de los animales (elementos tales como objetos, sombras, brillos, ruidos o personas que distraen o asustan al ganado) y la capacitación del personal (Grandin, 1998; 2000; Gallo y col, 2003 a)

El bienestar de los animales durante el manejo se puede medir a través de indicadores de estrés en sangre (indicadores fisiológicos), determinando variables tales como: cortisol, glucosa, hematocrito, lactato, creatinfosfoquinasa antes y después de someter a los animales a los distintos manejos (Shaw y Tume, 1992).

Además de los indicadores fisiológicos, también es posible usar otros indicadores para evaluar el bienestar animal. Grandin (1998) plantea el uso del comportamiento animal como indicador de bienestar, y recomienda cuantificarlo determinando: el porcentaje de animales en que se usa picana eléctrica (se considera un máximo aceptable de 25\%); el porcentaje de animales que resbala durante estos manejos (máximo aceptable de 3\%), el porcentaje de animales que cae durante el arreo (máximo aceptable de 1\%) y el porcentaje de animales que vocaliza (muge en el caso de los bovinos) durante el mismo (máximo aceptable 3\%). Estos indicadores reflejan dificultades durante el avance y/o dolor en los animales; se usan en las auditorías que realiza el Instituto Americano de Carnes en USA para verificar cómo está el manejo y bienestar animal en las plantas faenadoras; sin embargo, es posible aplicarlos para evaluar el manejo en otras situaciones, tales como durante el arreo, carga y descarga a nivel productor, en las ferias ganaderas y otros lugares.

En cuanto al reposo posterior al transporte, en matadero, Tadich y col (manuscrito en arbitraje) señalan que el prolongar su duración (de 3 hasta 24 horas), con la finalidad de que las concentraciones de las variables sanguíneas recuperen los valores normales, no es 
una buena medida, ya que la recuperación lograda es escasa y no se justifica si se toma en cuenta los efectos negativos sobre la canal.

Los tiempos prolongados de privación de alimento, además de afectar los indicadores sanguíneos de estrés, pueden provocar pérdidas de peso que comprometen la canal, así como también alteraciones de la calidad de la carne. (Gallo y Gatica, 1995; Gallo y col, 2003 b).

La exposición de los animales a varias condiciones adversas a la vez, tales como: falta de alimento o agua, peligro, hambre, mezcla de animales de diferente procedencia, ambiente molesto, fatiga, calor, frío, luz, restricciones de espacio y otras, condicionan en los animales un estado de estrés que puede tener efectos sobre la calidad de la carne (Forrest y col., 1979).

La carne presenta una coloración oscura y un $\mathrm{pH}$ alto, anomalía que en el bovino se conoce como "corte oscuro" (darkcuttingbeef, Hood y Tarrant, 1980). El corte oscuro se relaciona directamente con una disminución de los niveles de glicógenomuscular y hepático (Gallo y Lizondo, 2000; cuadro 3), por lo cual el riesgo de problemas de calidad aumenta si los animales están mucho tiempo privados de alimento y además en ambientes extraños; así también tendrán mayor riesgo aquellos animales que traen una baja reserva de glucógeno inicial desde el predio (Mc Veigh y Tarrant, 1982).

Por lo anterior, entre los factores predisponentes de corte oscuro destaca una relación positiva con el tiempo de ayuno, siendo más evidente si el transporte previo también ha sido largo (Gallo y col., 2003) Consecuentemente se deberían evitar las esperas prolongadas de los animales, tanto en predios, ferias como mataderos, en particular de aquellos con transporte prolongado antes o después, y procurar que los reposos sean realmente un descanso y no un estrés adicional.

Es indudable que mientras más se prolongan las esperas, más eventos adversos pueden presentarse durante las mismas. Dado que las carnes con elevado pH son inaptas para el envasado al vacío, por su rápido deterioro, estas canales son castigadas en el precio, a pesar de que son sólo algunos músculos (cortes de carne) los afectados (Almonacid, 2003).

Durante el transporte de los animales, además de pérdidas de peso, se producen con frecuencia traumatismos, contusiones o daños físicos. Las contusiones están definidas en grados, de acuerdo a su profundidad aquellas de grado 1 afectan sólo el tejido subcutáneo, 
las de grado 2 afectan también el tejido muscular y las de grado 3, los tejidos subcutáneos, muscular y óseo. Las pérdidas económicas por cantidad de recortes y calidad de carne que se producen por el destino limitado que se les puede dar a las canales traumatizadas son considerables (Godoy y col.1986).

Durante el transporte no solo influye la duración del viaje sobre el bienestar de los animales, sino también las características del vehículo, del chofer y su conducción, las características de los caminos (curvas, pendientes, ripio, etc.), del clima y la temperatura ambiental (calor, frío, lluvia, nieve, etc.), las características de los animales transportados en particular (edad, sexo, presencia o no de cuernos, estado nutricional y sanidad), la densidad de carga y otros.

El objetivo de insensibilizar al animal antes de sangrarlo es lograr que éste pierda instantáneamente la consciencia y no la recupere antes de la sangría, de manera que no sienta dolor, se inmovilice y sea más fácil y seguro para el operario manejarlo. Entre los indicadores que se pueden usar para determinar los efectos de la insensibilización o noqueo sobre el bienestar animal están los fisiológicos, como los niveles sanguíneos de cortisol, glucosa y lactato medidos en el momento de la sangría (Tadich y col. (2002). Pero además de los indicadores fisiológicos, para determinar la eficacia de la insensibilización y evaluar el bienestar animal durante el proceso, Grandin (1998) recomienda usar algunos indicadores de comportamiento.

\section{Análisis Sensorial}

La evaluación sensorial es una disciplina científica utilizada para medir, analizar e interpretar respuestas a las propiedades de los alimentos por medio de los sentidos (vista, olfato, sabor, tacto y oído) (Hollander, 1998). Según Muñoz y Chambers (1993), la información hedónica que se obtiene es una herramienta valiosa porque provee información más en concordancia con la de los consumidores, que son los únicos que pueden indicar con veracidad el grado de aceptación o rechazo de un producto.

El color se considera una de las características sensoriales más importante en la apariencia de un alimento. Se determina por el largo de onda entre 380 y $770 \mathrm{~nm}$ y se puede definir como la energía radiante que el ojo humano detecta a través de sensaciones visuales recibidas por la estimulación de la retina (Kramer, 1976).

El color de la carne es el resultado de la presencia de dos pigmentos: mioglobina y hemoglobina. El contenido de mioglobina se utiliza como un indicador de color (Pearson, 
1966). Un sistema de colorimetría utilizado en la determinación de color en alimentos es el sistema Hunter. Este sistema también conocido como color uniforme está basado en la teoría de los colores oponentes a la visión de color. En esta teoría se asume que hay un estado de conexión-señal intermedia entre los receptores de luz en la retina y el nervio óptico que transmite las señales de color al cerebro. En este mecanismo de conexión, las respuestas al rojo son comparadas con verde y resulta en una dimensión de color de rojo a verde. Las respuestas al verde son comparadas con azul para dar una dimensión de color de amarillo a azul. Esas dos dimensiones son representadas por los símbolos de a y b. La tercera dimensión de color es la luminosidad o blancura expresada como L (deMan, 1999). La escala de Hunter Lab es una de las más usadas ya que es fácil de interpretar.Utiliza tres parámetros: $\mathbf{L}, \mathbf{a}$ y $\mathbf{b}$, donde $\mathbf{L}$ mide las tonalidades de blanco (100) hasta negro (0), a mide las tonalidades de rojo (+) hasta verde (-) y b las de amarillo (+) hasta azul (-).

La calidad de la carne varía entre músculos dentro de la misma canal y entre réplicas debido a factores antemortemy postmortem; los cuales son complejos y difíciles de controlar (Rhodes, 1979). Varios factores pueden afectar el color de la carne cruda. Si al momento de la matanza del vacuno, el contenido de glicógeno en el tejido muscular es anormalmente bajo, la carne tiende a ser oscura al presentar una estructura compacta y absorber más luz. Ello es debido a que anaeróbicamente se produce poco ácido láctico y consecuentemente, el pH de la carne postmortemse mantiene más alto de lo normal (mayor o igual a 6) y como resultado, se acorta el tiempo de vida útil de la misma. Sin embargo, esta carne tiende a ser jugosa, tierna y con una excelente capacidad de retención de agua ("water holding capacity") (Kauffman,1993). Este fenómeno que ocurre en la carne se conoce como "Dark, Firm and Dry" y está asociado al estrés que sufre el animal al momento de la matanza, a factores hereditarios y estacionales (Pearson y Dutson, 1994).

Respecto a la carne bovina, producción tradicional en los países de Mercosur, se nota una demanda a nivel mundial cada vez mayor, pero acompañado de un estancamiento de los stocks de ganado durante los últimos 3 años. La FAO ha estimado que la producción de ganado bovino se mantuvo casi invariable en alrededor de 65 millones de toneladas entre 2008 y 2011, aunque el comercio de la carne vacuna se ha incrementado en un $2 \%$, hasta 7.7 millones de toneladas de carne en 2011, a partir del crecimiento de la demanda en 
países que enfrentan un alto crecimiento económico y/o una caída de la producción (FAO 2011).

El Mercosur es uno de los principales agentes dentro de la industria cárnica a nivel mundial, especialmente en la carne bovina, donde los cuatro países son importantes productores, consumidores y exportadores. Las regiones competidoras (Estados Unidos, Unión Europea, Oceanía) están mostrando diferentes problemas para aumentar e incluso mantener los niveles de producción que gozaban en el pasado (USDA ForeignAgriculturalService, 2011).

Este crecimiento dispar entre los países de Mercosur tiene buena parte de su origen en eventos internos a los países. Por un lado, Brasil cuenta con una política de incentivos para el fomento de la producción local, lo cual se complementa con la creación de grandes jugadores internacionales de origen brasileño para la competencia en el mercado internacional. El Uruguay, por su parte, tiene una gran tradición en la exportación de carne, la cual ha tenido una importante recuperación en los últimos años.

Este fenómeno se extendió también al consumo interno: se ha convertido en el país con mayor consumo per cápita de carne en el mundo (Webber 2011). Argentina, a pesar de la leve recuperación en los últimos 2 años, ha sufrido una baja de su participación en los mercados mundiales debido a la falta de políticas públicas coordinadas para la mejora de la producción ganadera y al tipo de controles gubernamentales ejercidos sobre la exportación de carne; tales situaciones han imposibilitado el aprovechamiento de las favorables condiciones actuales para la venta de carne. Tanto Uruguay como Paraguay han aprovechado el retiro de Argentina de ciertos mercados para aumentar sus exportaciones. Sin embargo, este aprovechamiento fue limitado debido al reducido stock vacuno en ambos países (USDA ForeignAgriculturalService, 2011).

El crecimiento del Paraguay como exportador de carne bovina es una de las características más notables de este proceso. Paraguay ha basado su exportación en la mejora sustancial de la genética animal y un sostenido esfuerzo para la entrada en nuevos mercados. Si bien los valores de exportación de Paraguay son menores a los de Argentina y Brasil, el primero ha experimentado un importante salto en cuanto a la exportación respecto a estos países, teniendo el crecimiento más rápido de la región.

El sector cárnico tiene una gran tradición en el Paraguay: es uno de los sectores productivos más antiguos del país. No obstante, históricamente ha sido considerado un 
producto para el mercado interno, debido a su calidad. Para 1899 el stock de ganado vacuno se estimaba en cerca de 2.200 .000 cabezas, y las exportaciones de cueros empezaron a jugar un rol más importante en la generación de divisas. Para 1915, el total del stock de ganado estaba alrededor de los 4 millones de cabezas (cfr. HerkenKrauer, Arce, y Ovando 2011). A lo largo del siglo XX, para Paraguay la carne ha tenido un desempeño exportador de menor porte que el cuero.

Además de los códigos sobre inspección de la carne, la Comisión del Codex Alimentarius ha elaborado el Código Internacional Recomendado de Prácticas de Higiene para la Carne Fresca (CAC/RCP 11-1976 Rev. 1-1993) y el Código Internacional Recomendado de Prácticas de Higiene para la Elaboración de la Carne de Aves de Corral (CAC/RCP 141976), en los que se contemplan los requisitos higiénicos mínimos para la producción inocua de carne fresca y carne de aves de corral.

Las disposiciones de la Comisión del Codex Alimentarius incluyen directrices sobre la construcción de los mataderos y demás instalaciones necesarias, control de plagas, calidad del agua para limpieza y desinfección, normas sobre inspección de la carne y prácticas higiénicas (incluida la supervisión por un inspector veterinario).

Con la necesidad de integrar en un solo organismo las actividades relativas a la salud animal y salud pública a fin de cubrir toda la cadena de producción y brindar mayor cobertura, eficiencia y confiabilidad a los servicios prestados por la Institución, llevo a la promulgación el 28 de julio del 2004 de la Ley $\mathrm{N}^{\circ} 2.426$, por la cual se crea el Servicio Nacional de Calidad y Salud Animal (SENACSA). Ente autárquico y autónomo que unifica el Servicio Pecuario Oficial del Paraguay integrando al Servicio Nacional de Salud Animal., creado por Ley No 99/91 y las Direcciones de Protección Pecuaria (DPP) y Normas de Control de Alimentos de Origen Animal (DINOCOA) del Viceministerio de Ganadería creadas por Ley 81/92, con la visión de servicio pecuario oficial promoviendo la competitividad del sector para el desarrollo; y la misión de apoyar la política pecuaria nacional, de incrementar la competitividad, la sostenibilidad y la equidad, de fomentar la productividad con calidad animal, fomentando el desarrollo económico con responsabilidad social y medio ambiental (BACCN, 2012).

El gobierno, que incluye a los servicios veterinarios gubernamentales, juega un papel clave en la higiene de la carne. Este papel será administrado por una autoridad competente que cumple un número de funciones esenciales. Una autoridad competente se define 
como: la autoridad oficial encargada por el gobierno del control de la higiene de la carne, incluyendo el establecimiento y la aplicación de requisitos regulatorios de la carne (FAO /OMS, 2004 ${ }^{\mathrm{a}}$ )

Las actividades de higiene de la carne son usualmente operadas por una autoridad competente que debe de proveer del número suficiente de personal capacitado para realizar dichas tareas. Los recursos que se requieren para realizar las tareas higiénicas incluyen equipo, trasporte, programas de entrenamiento y laboratorios.

Todos los procedimientos de inspección y su dictamen deben de ser realizados por personal competente. El apoyo del laboratorio es esencial para realizar la higiene de la carne, los laboratorios deben de ser evaluados y / o acreditados bajo programas oficialmente reconocidos, para asegurar que existan adecuados controles de calidad y metodologías válidas. La operación de servicios de higiene debe incluir la retroalimentación apropiada de la información a través de la cadena de producción del alimento, con especial atención, en regresar la información de la inspección a los productores.

Los certificados de salud internacionales que proveen garantía oficial para el comercio de las carnes deben de generar absoluta confianza en el país de importación (FAO/OMS, 1995). Los países importadores tomaran medidas proporcionales para la verificación de los certificados, por ejemplo, revisiones físicas y de documentos en los puertos de entrada y auditorias de terceros a los sistemas de higiene en el país exportador.

La vigilancia de la salud animal constituye una investigación continua de una población dada para detectar la ocurrencia de enfermedad para propósitos de control, y el monitoreo implica programas dirigidos a la detección de cambios, en la prevalencia de una enfermedad de una población dada, (OIE, 2004)

El acuerdo de Medidas Sanitarias y Fitosanitarias (SPS), Y Obstáculos Técnicos al Comercio (TBT) de la Organización Mundial del Comercio (WTO) representan los mejores esfuerzos de la comunidad global para establecer principios, y lineamientos que gobiernen las medidas para alimentos en el comercio internacional, la firma del acuerdo SPS en 1994 ha fomentado las medidas de higiene de la carne basadas en una evaluación general de los riesgos a la salud, humana y animal. 


\section{ESTRATEGIAS METODOLÓGICAS O MATERIALES Y MÉTODOS}

El estudio se desarrolló en la ciudad de Pilar, Departamento de Ñeembucú, específicamente con las empresas o comercios dedicados al rubro de venta de productos cárnicos, en el año 2017.

Es un trabajo descriptivo, que proporciona un perfil detallado del fenómeno de estudio, "La investigación descriptiva es aquella que busca especificar las propiedades, características, y los perfiles importantes de personas, grupos, comunidades o cualquier otro fenómeno que se someta a un análisis" (Danhke, 1989). En este sentido, con el presente trabajo se pretende describir las condiciones higiénico-sanitarias del proceso de transporte y comercialización de la carne vacuna en la ciudad de Pilar, en el año 2017.

Adoptando, el enfoque del tipo mixto, el método cuantitativo que busca obtener informaciones referentes a los conocimientos básicos relacionados al tema de análisis. El enfoque cualitativo permite levantar datos mediante entrevistas hechas a propietarios de carnicerías y a informantes claves, con el objeto de recoger informaciones que permitirán describir las percepciones, ideas y pensamientos relacionados al tema de la investigación. Las fuentes de datos de la misma, son: como primarias: Comercios de expendios de productos cárnicos, Municipalidad de la ciudad de Pilar, y como fuentes secundarias: las Legislaciones Nacionales, Legislaciones comparadas y las Disposiciones municipales.

La población se compone por 64 carnicerías de la ciudad de Pilar, registradas y habilitadas por la Municipalidad local; además de informantes calificados como los responsables del Área de control de calidad de la Municipalidad de la ciudad de Pilar y del SENACSA. La muestra seleccionada en la elección al azar del 30\% de las carnicerías de Pilar, asciende a 20 negocios. Además de los responsables del área de Control de calidad de la Municipalidad local y el SENACSA de la ciudad de Pilar.

Las técnicas implementadas para la recolección de los datos fueron: el Cuestionario: Administrado a los propietarios de las carnicerías de la ciudad de Pilar, una Entrevista semiestructurada a Informantes calificados: realizadas a los informantes calificados tales como responsables del control de calidad de la Municipalidad local y representantes del SENACSA de la ciudad de Pilar, además del Análisis Documental, con la revisión de registros históricos respecto a la calidad de la carne bovina en la ciudad de Pilar, que fueron solicitados y extraídos de los entes encargados de la calidad y sanidad animal; así 
también la Observación directa: Técnica relevante para corroborar la descripción del fenómeno estudiado.

\section{RESULTADOS Y DISCUSIÓN}

El cuestionario arrojó los siguientes resultados.

De los propietarios encuestados, el $10 \%$ respondió que su permanencia en el mercado es menor a un año, el 55\% afirma que su permanencia es de entre 1 año y 5 años, y el $35 \%$ restante se encuentra en el mercado con una antigüedad de más de 5 años.

La situación de tenencia del salón comercial, según el $65 \%$ son alquiladas, el 10\% equivale a salones prestados y el $25 \%$ restante corresponde a salones propios.

Según lo referido, se puede observar que el $95 \%$ de los propietarios cuentan con la habilitación correspondiente por la Municipalidad de Pilar, y el $5 \%$ restante se encuentra en proceso de gestión.

El $60 \%$ de los encuestados menciono que adquiere la carne bovina para su comercialización de la Ciudad de Pilar, mientras que el 25\% afirma que adquiere carne de importación y el $15 \%$ restante de otros puntos del país.

Los encuestados mencionaron que el volumen promedio de comercialización es de 1800 a $2000 \mathrm{~kg}$. Al mes. En relación a la frecuencia de adquisición de la carne bovina para su posterior comercialización, el $80 \%$ de los propietarios de las carnicerías contestó que lo realiza de forma semanal, el $10 \%$ con frecuencia quincenal y el 5\% restante adquiere dicho producto para su venta con una frecuencia mensual.

Según lo expresado por el $80 \%$ de los encargados y/o propietarios de las carnicerías conocen mucho a cerca de las disposiciones higiénicas sanitarias del trasporte y comercialización de la carne vacuna, mientras que el 15\% menciona conocer poco y el $5 \%$ restante no conoce; el $75 \%$ de los encuestados mencionaron que institución encargada de realizar los controles de calidad de la carne que expende en su negocio es la Municipalidad de Pilar, mientras que el 25\% afirma que ninguna institución controla su negocio.

El $75 \%$ de los encuestados mencionaron que solo a veces reciben a inspectores de la Municipalidad para realizar los controles correspondientes, mientras que el $25 \%$ afirma que nunca son visitados por dichos inspectores.

Para mantener la calidad de la carne vacuna realizo una limpieza profunda en mis equipos. generalmente realizo mantenimiento adecuado de mis equipos de refrigeración, realizar 
limpieza de la carne, capacitar al personal para que cuide esos aspectos; la mayoría de los encuestados coincidieron en que realizan una limpieza profunda y mantenimiento de los equipos para mantener la calidad de la carne.

Las características de las instalaciones, equipos y otras características que posee la comercialización de la carne bovina: Salón comercial (dimensión, materiales utilizados para la construcción: 5x3,10×8 salón loza, 6x5 material cocido, 4x4 material cocido, mampara, 6x6 salón loza. En la cual, la mayoría de los encuestados mencionaron tener un salón de material cocido y loza.

Con respecto a tenencia y disposición de equipos (heladeras, freezer exhibidores sierras, aire acondicionado) los encuestados mencionaron: Poseer todos los equipos mencionados y también un generador para los cortes de energía eléctrica, No poseer aire acondicionado, sólo dos ventiladores grandes. No poseer exhibidores, pero si contamos con los demás equipos. No poseer generador, pero contamos con aire acondicionado, sierra y congeladores.

En relación a la utilización de equipos para la realización de los cortes y la atención del cliente (gorros, delantales, guantes) manifestaron, que, no utiliza ninguno de esos elementos, si utiliza delantal y guantes para brindar una seguridad de higiene a mis clientes, solo utiliza delantal para no ensuciar mi ropa y tener un aspecto más higiénico, utilizo los equipos cuando recibo la carne, solo utilizo delantal.

De la entrevista a Informantes Calificados, se obtiene los siguientes resultados (funcionarios del Dpto. de Control de Calidad de la Municipalidad de Pilar).

Los requisitos para la habilitación de una carnicería en la ciudad de Pilar, son los mismos que se exigen a otros negocios dedicados al comercio, sin embargo, para el caso de expendio de carne de todo tipo, sean estos de ganado menor (peces, aves, porcino, ovino, caprino, etc.) y también el bovino; se debe poseer principalmente de congeladoras para su conservación, de modo a no perder la cadena de frío.

Al igual de otros negocios, las carnicerías pagan un impuesto pos su habilitación y un canon de forma anual.

Las condiciones higiénico/sanitarias para la comercialización de carne vacuna en las carnicerías de la ciudad de Pilar. Se establece que la carne faenada deberá permanecer por lo menos una hora colgada al gancho y previa autorización del veterinario se trasladará a los puntos de venta (Ordenanza Municipal $\mathrm{N}^{\circ}$ 50/99). Teniendo en cuenta 
que la carne bovina no apta para el consumo, puede ser propagadora de varias enfermedades transmisibles al ser humano (Zoonosis). Es por ello que el estricto control sanitario es de exclusiva responsabilidad del profesional Veterinario, funcionario de la Municipalidad, quien autoriza la comercialización de la carne, previamente inspeccionada. Por otro lado, el estricto control del origen legal de los animales a faenarse, será de exclusiva responsabilidad del encargado o Comisario de Tablada del Matadero. Para comercializar la carne vacuna en Pilar, el salón deberá estar azulejado, como mínimo a 2 metros de altura, debe estar limpio y desinfectado todo el tiempo.

Según el Art $\mathrm{N}^{\circ} 5$ de la Ordenanza Municipal $\mathrm{N}^{\circ}$ 50/99 "POR LA CUAL SE REGLAMENTA EL FAENAMIENTO Y COMERCIALIZACIÓN DE CARNE PARA EL CONSUMO PÚBLICO”, menciona que: La venta de carne al público se hará únicamente en los puestos establecidos y habilitados para el efecto.

Las reses faenadas en otros distritos, solamente serán comercializados en la misma y en ningún caso serán introducidas en el Municipio de Pilar para la venta.

Sin embargo, La Ley 3966/2010 de la Carta Orgánica Municipal, que contempla la Ordenanza Municipal № 111/215, en su Art. 14.5 trata sobre el "Ingreso de las carnes provenientes de otros Municipios”, menciona que: Las carnes de otros municipios deberán ser transportadas de acuerdo a las exigencias sanitarias requeridas por la ley y la ordenanza municipal.

Los propietarios deberán presentar la certificación del Veterinario de la Matadería o Frigorífico de origen. Los responsables y/o dueños deberán abonar por cada kilo de carne la suma de Gs. 1.000 (guaraníes mil), en concepto de inspección sanitaria. De manera constante se realiza la inspección a las carnicerías, y la carne deberá estar sellada por la matadería. Deben poseer buena apariencia, de procedencia confiable, sin olores desagradables, y por, sobre todo, debe llevar el sello de calidad de la Matadería donde fue faenado el animal.

Se debe cumplir con la Ordenanza Municipal No 50/99 "Por la cual se reglamenta el faenamiento y comercialización de carne para el consumo Público"; también la Ordenanza Tributaria Municipal $\mathrm{N}^{\circ} 111 / 215$, en la cual menciona los aranceles a ser abonados para las faenas y las documentaciones pertinentes de las cuales deben ir númidas las personas que se dedican a esta actividad. 
En general se puede mencionar que es recular la situación del transporte y comercialización de carne vacuna en la ciudad de Pilar, haciendo la salvedad de que falta un mayor control del ingreso y procedencia de las carnes comercializadas en determinadas carnicerías. Ya que se han dado casos de reses introducidas al mercado local, de dudosa procedencia y sin los permisos y habilitaciones necesarias para su consumo al público.

\section{CONCLUSIÓN O CONSIDERACIONES FINALES}

Una vez analizado los resultados obtenidos en la investigación, y basados en los objetivos específicos propuestos inicialmente, a continuación, se presentan las siguientes conclusiones:

\section{Disposiciones legales previstas por los Organismos de control de calidad en el proceso de transporte y comercialización de la carne vacuna en la ciudad de Pilar.}

Las disposiciones legales que regulan la faena, transporte y comercialización de carne en la ciudad de Pilar y alrededores, son aquellas contempladas en la Ordenanza Municipal $\mathrm{N}^{\circ} 50 / 99$ "Por la cual se reglamenta el faenamiento y comercialización de carne para el consumo Público"; también la Ordenanza Tributaria Municipal N 111/215, en la cual menciona los aranceles a ser abonados para las faenas y las documentaciones pertinentes de las cuales deben ir númidas las personas que se dedican a esta actividad.

Además, el SENACSA (Servicio Nacional de Calidad y Salud Animal), es el ente encargado de la provisión de los permisos pertinentes a propietarios de carnicerías, con la cual autoriza el expendio y comercialización de carne bovina en el mercado local.

Características físicas y sensoriales que presentan las carnes que se expenden en el mercado de la ciudad de Pilar.

Debemos tener en cuenta y estar muy claros que la carne se debe consumir madura, de esa manera representaría efectos secundarios en nuestra salud. La carne está constituida por tejido muscular y grasa intermuscular los cuales son muchos. La clasificación de la carne según la grasa que contiene la medimos en porcentaje de la siguiente manera:

- CARNE MAGRA: Cuando el contenido de grasa en menor al $14 \%$

- CARNE SEMIGORDA: El contenido de grasa varía entre el $14 \%$ y $20 \%$

- CARNE GORDA: El contenido de grasa varía entre $20 \%$ y $30 \%$

- CARNE MUY GORDA: El contenido supera el 30\% 
De acuerdo a las observaciones realizadas, las características físicas de la carne se determinan por los sentidos del olfato, la vista y el tacto. Tal es así que, en general, las carnes comercializadas en las carnicerías de Pilar y alrededores poseen aspectos imperceptibles en cuanto al olor, salvo algunas pequeñas carnicerías que generan ciertos olores desagradables por los restos de carne y hueso que han quedado por las cierras, serruchos y cuchillos. El aspecto general en cuanto a la percepción visual, se observan carnes de mediana a buena calidad, ya que en muchos casos estas provienen de animales viejos o vacas de descarte, por lo que generalmente la carne resultante no es del todo tierna.

\section{Mecanismos de control establecidos para la verificación de la calidad de los productos ofrecidos al público por parte de las Carnicerías de la ciudad de Pilar.}

Según expresiones del Inspector General de la Municipalidad de Pilar, las inspecciones de la calidad de la carne se realizan de manera constante en las diferentes carnicerías, sin embargo, muchos propietarios de carnicerías aseveraron que dichos controles no son constantes y que generalmente el personal de la Municipalidad se acerca a sus negocios para cobrar los impuestos respectivos que conllevan dicha actividad.

Por otro lado, según la Ordenanza Municipal N 50/99 "Por la cual se reglamenta el faenamiento y comercialización de carne para el consumo Público", menciona que el estricto control sanitario es de exclusiva responsabilidad del profesional Veterinario, funcionario de la Municipalidad, quien autoriza la comercialización de la carne, previamente inspeccionada. Por otro lado, el estricto control del origen legal de los animales a faenarse, será de exclusiva responsabilidad del encargado o Comisario de Tablada del Matadero.

\section{LISTA DE REFERENCIAS}

Asociación Rural del Paraguay. (2017). Paraguay y su Sector Cárnico. Asociación Rural del Paraguay, 1 - 12. Obtenido de https://www.arp.org.py/images/Paraguay-y-elSector-Carnico.pdf

BACCN. (Diciembre de 2012). Ley No 2426 / CREA SERVICIO NACIONAL DE CALIDAD Y SALUD ANIMAL (SENACSA). BACCN - Leyes Paraguayas., 1 17. Obtenido de https://www.bacn.gov.py/leyes-paraguayas/365/ley-n-2426crea-servicio-nacional-de-calidad-y-salud-animal-senacsa

BROWN, Richard. 2010. «Long Term trends in World Meat Trade 2010-2015-2020». 
FAO (2007). Manual. Buenas prácticas para la industria de la carne. Producción y sanidad animal. ISBN: 1810-1143. Roma.

FRASINELLI, C. A., Casagrande, H. J. y Veneciano, J. H. 2004. INTA E.E.A San Luis, Información Técnica 168, 16.

Gallo, C., \& Tadich B., N. (octubre de 2008). Bienestar animal y calidad de carne durante los manejos previos al faenamiento en bovinos. Revista Electrónica de $\begin{array}{llllll}\text { Veterinaria, } & \text { IX, } & 1 & - & 19 . & \text { Obtenido de }\end{array}$ https://www.redalyc.org/pdf/636/63617111001.pdf

GALLO, C. 1994. Efecto del manejo pre y post faenamiento en la calidad de la carne. Serie Simposios y Compendios de la Sociedad Chilena de Producción Animal vol.2: $27-47$

GARTZ Richart. Las carnes y su procesamiento 1987 almonacid, M. ALMONACID, M. 2003. Estudio de pH y color muscular en cortes comerciales de canales bovinas normales y con la anomalía "corte oscuro". Memoria de Título, Medicina Veterinaria, Fac. Cs. Vet. Univ. Austral de Chile, Valdivia, Chile.

Grupo Latino Ltda. (2006). Manual del Ingeniero de Alimentos. Colombia: Libro Digital Grupo Latino Ltda. Obtenido de http://repositoriodigital.tuxtla.tecnm.mx/xmlui/bitstream/handle/123456789/120 2/MANUAL\%20DEL\%20INGENIERO\%28Digital\%29.pdf?sequence=1\&isAll owed $=\mathrm{y}$

GUGLIELMONE, a.a. 1994. Epidemiología y control de los hemoparásitos (Babesia y Anaplasma) de los vacunos en la Argentina. In: Enfermedades parasitarias de importancia económica en la Argentina y el Uruguay. Eds.C. Fiel y A.Nari. Editorial Hemisferio Sur,Montevideo, pp. 461- 479.

HERKEN Krauer, Juan Carlos, Lucas Arce, y Fernando Ovando. 2011. «La Economía del Paraguay entre 1940-2008: Crecimiento, Convergencia Regional e Incertidumbres». En Economía y Estado en Paraguay. 1870-2010, eds. Fernando Masi y Dionisio (comp.) Borda. Asunción, Paraguay: CADEP.

Hernández Aliaga, E. C. (2009). Control de calidad de la carne: Métodos y Procesos. Lima - Perú: Universidad Nacional de Educación Enrique Guzmán y Valle. Facultad de Agropecuaria y Nutrición. Recuperado el 10 de enero de 2022, de https://es.scribd.com/doc/20402570/Calidad-Carne-metodos-y-Procesos 
Horcada, A. (Marzo - Abril. de 2000). La calidad de la canal y de La calidad de la canal $\mathrm{y}$ de la car la carne de $\mathrm{v}$ ne de vacuno en acuno en Navarra. NAVARRA AGRARIA, 56 - 64. Obtenido de file:///D:/Usuario/Downloads/carne.pdf

Instituto de Nutrición de Centro América y Panamá. (3 de marzo de 2020). Análisis Sensorial para control de calidad de los alimentos. Obtenido de Sistema de la Integración Centroamericana: http://www.incap.int/index.php/es/noticias/201analisis-sensorial-para-control-de-calidad-de-los-alimentos

PEEA-UCA. 2005. 148 Lineamientos para la Formulación de Escenarios del Mercado de la Carne Vacuna en Argentina. Buenos Aires, Argentina: IPCVA. Documento de Trabajo.

San Román, D. (2015). Características físicas de la Carne Natural del Paraguay. ASOCIACION RURAL DEL PARAGUAY FUNDACIÓN SOLIDARIDAD LATINOAMERICANA, 1 - $65 . \quad$ Obtenido de https://www.arp.org.py/images/files/Caracteristicas\%20Fisicas\%20de\%201a\%20 Carne\%20Natural.pdf

SERVÍN, Maria Belén. 2011. «Caracterizando al sector cárnico del Paraguay». Observatorio Económico de la Red Mercosur.

Tovar, J. (martes 26 de noviembre de 2019). ¿Qué contiene la carne? Propiedades Nutricionales . Obtenido de EFE:SALUD: https://www.efesalud.com/propiedades-nutricionales-carne/

Unidad Técnica de Estudios para la Industria. (Febrero de 2008). Industria de la Carne Bovina. Unidad Técnica de Estudios para la Industria, 1 - 4. Obtenido de https://www.mic.gov.py/mic/w/industria/deei/pdf/Perfil_Sect/Carne2008.pdf

http://www.produccion-animal.com.ar/informacion_tecnica/carne_y_subproductos/63calidad_carne.pdf

http://www.iica.org.py/observatorio/producto-paraguay-bovino-presentacion.htm http://www.entrevistadetrabajo.org/entrevista-mixta-o-semiestructurada.html www.monografias.com/trabajos81/tablas-preguntas-conceptos-es\&hellip http://www.senacsa.gov.py 